\title{
Impact of Optical Booster to Enhance the Gain Bandwidth for Dense Optical Communication System
}

\author{
Ghanendra Kumar ${ }^{1}$ and Chakresh Kumar ${ }^{2 *}$ \\ ${ }^{1}$ Department of Electronics and Communication Engineering \\ National Institute of Technology, Delhi-110040, India \\ ${ }^{2}$ University School of Information, Communication \& Technology, \\ Guru Gobind Singh Indraprastha University, New Delhi-110078, India \\ E-mail: *chakreshk@gmail.com
}

\begin{abstract}
This paper explains how the combination of RAMAN amplifier and PARA-METRIC amplifier using non-linear fiber components increases the gain bandwidth. Two kinds of configuration of amplifier are demonstrated with gain associated and bit error rate is seen. $15 \mathrm{~dB}$ gain is measured and flat gain of $5 \mathrm{~dB}$ is measured.
\end{abstract}

Keywords: FWM, OPC, RZ, NRZ, DWDM, OBP, XPM, EDFA, Mach-Zehnder Modulator

\section{INTRODUCTION}

In recent years, the most attracting technology is the RAMAN amplifier, which allows high-capacity data signals for long distance in the optical fiber communication. RAMAN amplifying device is in the research process due to its small size and high compact high energy pumping laser availability. Thus, both non-linear process fiber RAMAN Amplifier (FRA) and Optical PARA-METRIC Amplifier (OPA) hybrid is used to develop wide bandwidth amplifying device [1-5]. RAMAN amplification technology demonstrates the increase in transmitting capacity and span length. FRA output showed results of ultra-wide BW, lower noise and suppressing nonlinearity. Discrete RAMAN Amplifier (DRA) using Dispersion compensating fiber (DCF)/Highly non-linear fiber (HNLF) have good noise output and better signal budget. PARAMETRIC amplifier uses highly productive four-wave mixing in fiber optics. We can get a gain value around $49 \mathrm{~dB}$ and bandwidth of $200 \mathrm{~nm}$ by proper design of HNLF [6]. The demand of large bandwidth, less noise figure and also less gain ripple values makes both FRA's and OPA's more attractive over tradition EDFA [7]. Studies have concluded that RAMAN assisted EDFA having configuration of hybrid yielding low noise figure (NF) and optical to signal ratio. Flat gain characteristics over $\mathrm{C}$ and $\mathrm{L}$ band are used in RAMAN/ EDFA hybrid amplifier. But, bandwidth gets restricted by Er ions. FRA design laser problem of quandary of pump interaction. A lot of compact amplifier are used the minimum of noise signal or gain fluctuation will be multiplied several times in te end. E Golo Vehecnko, Hsieh and Freitas have tried to increase gain-enhancing effect on the coupling of two non-linear process. In this paper we see a way of combining FRA and HNLF to achieve gain around or over $15 \mathrm{~dB}$ and a gain ripple within $5 \mathrm{~dB}$ [8-12].

\section{MATHEMATICAL MODEL}

Mathematical model is divide into two parts-A. Power Pump evalutation for co- propagation para-metric and counter propagating RAMAN pump. B. Net signal gain A. If both the amplifier interact using RAMAN effect, thus the coupling equation used in RAMAN effect can be represented as

$$
\begin{aligned}
& d P / d z=g \lambda R / A_{\text {eff }} * P_{R} P_{P}-\&_{p} P_{P} \\
& d P_{R} / d z *=-w_{R} / w_{P} A_{\text {eff }} * g \lambda R P_{R} P_{P}-\&_{R} P_{R} \\
& A_{\text {eff- }} \text { Effective mode area } \\
& P_{R}-\text { RAMAN effective power } \\
& P_{P}-\text { PARA-METRIC effective power, } \&_{R}-\text { Attenuation }
\end{aligned}
$$
coefficient (RAMAN), $\&_{p^{-}}$Attenuation coefficient (PARA-METRIC), Thus, initially $\mathrm{P}_{\mathrm{P}}(\mathrm{l}=0)=\mathrm{P}_{\mathrm{P} @ 0}[\mathrm{w}]$ while $\mathrm{P}_{\mathrm{P}}$ $(\mathrm{L} \mathrm{km})=\mathrm{P}_{\mathrm{R} @ \mathrm{~L}}[\mathrm{~W}]$ 


\section{Impact of Optical Booster to Enhance the Gain Bandwidth}

Now the solution can be obtained by dividing the wire into small lengths of delta $\mathrm{z}$ with constant light power. Also iterating using fourth order Range- Kutta method power output can be calculated.

B. The net gain by RAMAN pump and the PARA-METRIC pump are represented by

$$
\begin{aligned}
& g_{R}(\lambda, L)=\left[g_{R}\left(\lambda, \beta_{1}\right) g_{R}\left(\lambda, \beta_{2}\right) \ldots . . g_{R}\left(\lambda, \beta_{n}\right)\right] \\
& g_{P}(\lambda, L)=\left[g_{P}\left(\lambda, \beta_{1}\right) g_{P}\left(\lambda, \beta_{2}\right) \ldots . . g_{p}\left(\lambda, \beta_{n}\right)\right]
\end{aligned}
$$

Where $g_{R}(\lambda, L)$ and $g_{p}(\lambda, L)$ are gain of each segment in both the pumps respectively.

\section{RAMAN GAIN IN EACH SEgMENT}

The pump power level may be derived as

$$
\begin{aligned}
& \mathrm{dP}_{\mathrm{s}} / \mathrm{dz}=\mathrm{g} \lambda \mathrm{R} / \mathrm{A}_{\text {eff }} * \mathrm{P}_{\mathrm{R}} \mathrm{P}_{\mathrm{s}}-\&_{\mathrm{s}} \mathrm{P}_{\mathrm{s}} \\
& \mathrm{dP}_{\mathrm{R}} / d z^{*}=-\&_{\mathrm{R}} \mathrm{P}_{\mathrm{R}}
\end{aligned}
$$

Where, $\mathrm{P}_{\mathrm{S}}$ is power output by the signal. Thus, solving this equation using the small valued gain assumption over delta $\mathrm{z}$ length gives the total gain as-

$$
\left.-\&_{S} P_{S}\right)
$$

$g_{R}(\lambda$, delta $z)=\operatorname{EXP}\left[g_{R} P_{R}(\right.$ delta $z) / A_{\text {eff }} \&_{R}^{*}\left(e^{\&} R^{\text {delta }}-1\right)$

\section{Calculating PARA-METRIC Gain in Each SEGMENT (DELTA Z)}

According to small signal theory, there is a PARA-METRIC gain which amplifies the signal with idler rising at $w p=2 w p+w s$.

Therefore, small power gain at the length delta $\mathrm{z}$

$\mathrm{g}_{\mathrm{p}}(\lambda$, delta $\mathrm{z})=1+\left[\lambda \mathrm{P}_{\mathrm{p}}(\boldsymbol{\delta} \mathrm{z}) / \mathrm{g}_{\mathrm{p}} \sinh (\mathrm{g} \boldsymbol{\delta} \mathrm{z})\right]$

The PARA-METRIC gain $g$ is given by,

$\mathrm{g}^{2}=-\boldsymbol{\Delta} \mathrm{k}_{\mathrm{o}}\left(\boldsymbol{\Delta} \boldsymbol{k}_{\mathrm{o}} / 4+\boldsymbol{\gamma} \mathrm{P}_{\mathrm{p}}(\boldsymbol{\delta} \mathrm{z})\right]$

$\boldsymbol{\Delta} \boldsymbol{k}_{\mathrm{o}}$ is linear phase mismatch.

$$
\boldsymbol{\Delta} \boldsymbol{k}_{\mathrm{o}}=-2 \pi \mathrm{c} /\left(\lambda_{\mathrm{o}}\right)^{2} * \mathrm{dD} / \mathrm{d} \lambda\left(\lambda_{\mathrm{P}}-\lambda_{0}\right)\left(\lambda_{\mathrm{p}-} \lambda_{\mathrm{s}}\right)^{2}
$$

thus, the net gain is given by $\mathrm{G}_{\text {net, }}$

$G_{\text {net }}(\lambda, L)=10 \log \left[g_{R}(\lambda, L)+10 \log \left[g_{p}(\lambda, L)\right]\right.$
Gnet $=$ Net signal gain due to two pumps for the length (L)

$$
\begin{aligned}
& \mathrm{G}_{\mathrm{ON} / \mathrm{OFF}}=\operatorname{Gnet}((\lambda, \mathrm{L}))+\left|\mathrm{L}_{\mathrm{T}}\right| \\
& \mathrm{G}_{\mathrm{ON} / \mathrm{OFF}}=\text { on/off gain = sum of total component losses }
\end{aligned}
$$
(LT)

\section{EXPERIMENTAL SETUP}

By employing two different arrangements namely. Tandem and Unison, we observe the plausible gain BW increment of the amplifier. Fig.1 (a) shows the unison configuration. In this arrangement both the gains occur in the same HNLF. Para-metric pump source is a DFB laser $(\lambda=1603 \mathrm{~nm})$ which is a combination of $580-660 \mathrm{MHz} \mathrm{RF}$ frequencies centred at around $1603 \mathrm{~nm}$ frequency. Although a high power EDFA is used to amplify/ pump the para-metric pump (OPA), PARA-METRIC nonlinear interaction can be better generated by using high power LD with low noise. ASE noise is filtered by $1 \mathrm{~nm}$ wide passband filter. Signal source is a tunable laser whose power is kept constant at $-3 \mathrm{dBm}$. The modulation OSF, The signal beam was done using the LiNbo3 Mach Zehnder Dimension Modulator (MZM), which is powered by the enhanced output of a pattern generator, which produces 10 Gbps NRZ pseudo bit stream and bit stream behaviour is randomly. (PRBS: 10-31). The para-metric pump $(\lambda=1601)$ and the signal then went through a $1452 \mathrm{~nm} / \mathrm{C}$ and $\mathrm{L}$ band Wavelength Division multiplexer (WDM) before they were launched in $550 \mathrm{~m}$ long HNLF fiber. The HNLF that has a smallest dispersion wavelength $\lambda^{\prime}=1590 \mathrm{~nm}$, an effective area Aeff = 16 into 10-12 m2A, a loss occurs of 0.7 $\mathrm{dB} / \mathrm{km}$, length $\mathrm{L}=550 \mathrm{~m}$ and a non-linear coefficient $\gamma=5.7$ into 10-3W-1 m-1. A $1452 \mathrm{~nm} / \mathrm{C}+\mathrm{L}$ coupler connects to the HNLF, A counter-propelled RAMAN pump focuses on $1452 \mathrm{~nm}$, in which the full width of $2 \mathrm{~nm}$ is half the maximum (FWHM) bandwidth. The signal passes through an edge filter with $1 \mathrm{~dB}$ insertion loss for wavelength shorter than $1593 \mathrm{~nm}$ and level of blocking is about $40 \mathrm{~dB}$ for wave-lengths greater than $1600 \mathrm{~nm}$. The signal is analyzed, when Edge and $12822 \mathrm{AO} / \mathrm{E}$ convert and use the sample oscilloscope to pass through the edge filter using the optical spectrum analyzer (OSA) in time and Wavelength's domain. BERT analyzer was used for bit error rate analysis. 


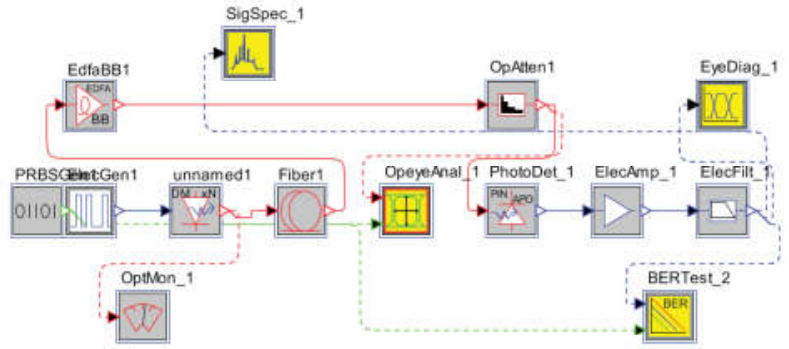

(a)

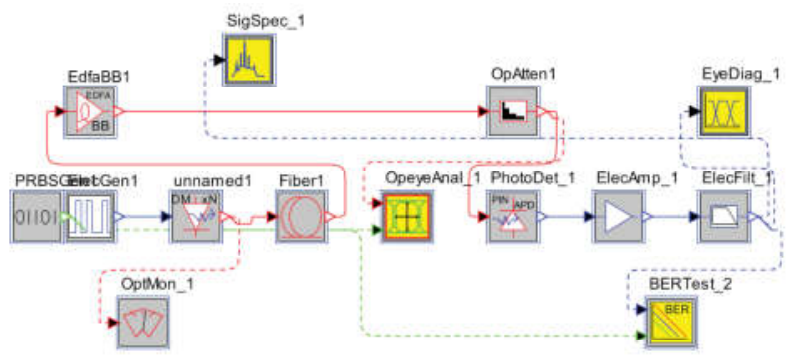

(b)

Fig. 1(a): Experimental Setup for Unison Structure of Hybrid RAMAN/Optical Fiber Amplifier, Figure 1(b): Exploratory Setup for Tandem Structure of the Hybrid Amplifier

Fig. 1(b) show the tandem configuration. Two separate 550nm long HNLF fiber are used for each of these processes. The signal and PARA-METRIC pump were coupled using 70/30 coupler, from where it is launched into the primary segment holding the PARA-METRIC process. At the end of this segment, edge filter allows the signal to pass through, neglecting $1603 \mathrm{~nm}$ signal. Two $1450 \mathrm{~nm} / \mathrm{C}+\mathrm{L}$ coupler holds the second segment. The second coupler joins the RAMAN pump to the HNLF, while the first dumps the RAMAN pump. Resulting signal which is allowed to pass through these couplers are observed for their spectral and temporal characteristics also error analyzer was used to give bit rate analysis.

\section{EXPLORATORY RESULT}

A. Ranging of the Gain Bandwidth $1^{\text {st }}$ independent individual characterisation of both the processes was observed. The on/off gain $\left(\mathrm{G}_{\mathrm{ON} / \mathrm{OFF}}\right)$ is defined as -

$$
\begin{aligned}
& G_{\text {ON/OFF }}-P_{\text {Sig_out(pump-on) }}-P_{\text {sig_out(pump- off) }} \\
& \text { Where, } P_{\text {sig_out(pump-on) }} \text { and } P_{\text {Sig_out(pump-off) }}
\end{aligned}
$$

Variables used are wavelength and power level of pumps. The wavelength is varied over $\mathrm{C}+\mathrm{L}$ band. For each of nonlinear pumps, two plots of the on/off gain curves were obtained against the variations.

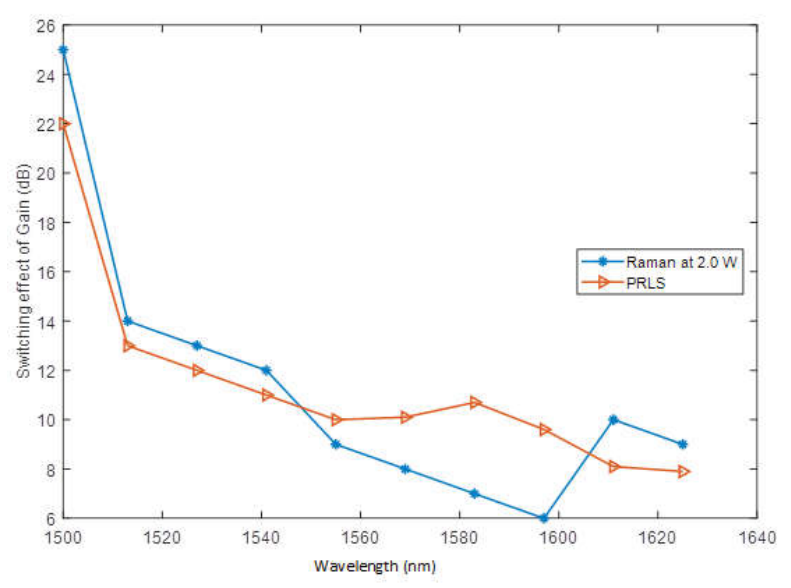

Fig. 2

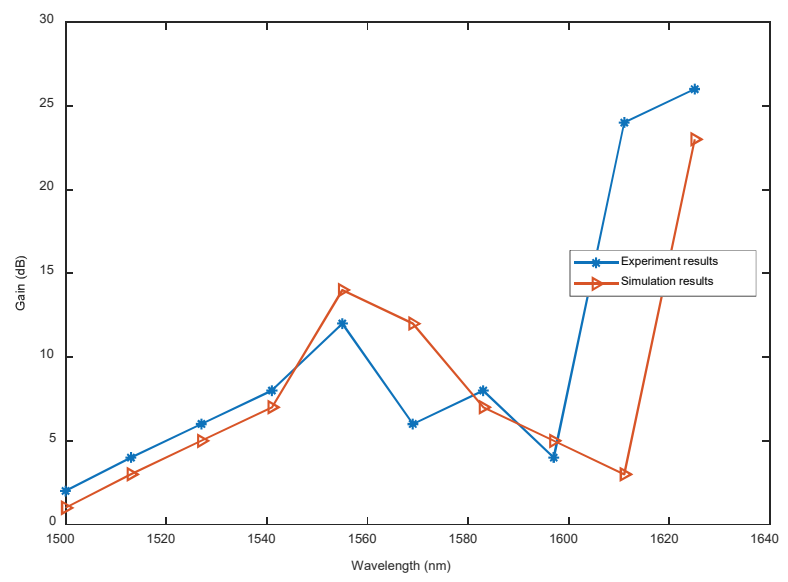

Fig. 3

Fig (2) and Fig (3) For RAMAN only pump at 1.6W, the on/off gain max $(15.2 \mathrm{~dB})$ was observed at $1555 \mathrm{~nm}$ and with increase with wavelength into $\mathrm{L}$, declined gradually. For PARA-METRIC, only pump the maximum (14.5dB) on/off gain was obtained at $1584 \mathrm{~nm}$. Now for hybrid effect, both pump were on with the same power level and variations of wavelength into $\mathrm{C}$ - $\mathrm{L}$ band. It was noted that there is a significant increase in the gain for the PARAMETRIC region while the gain decreases in the RAMAN region with the wavelength tuning from 1615 to $1580 \mathrm{~nm}$. The RAMAN dump port explains this phenomenon. It was found that when wavelength was coordinated with the RAMAN region, with RAMAN pump tune at $1.6 \mathrm{w}$ and PARAMETRIC pump at $0.69 \mathrm{w}$, there was about $15 \%$ of power transfer from the RAMAN pump power to the PARA-METRIC pump. This suggests that in UNISON configuration leads to power transfer from RAMAN pump to PARA-METRIC pump. Therefore, there is an increase in PARA-METRIC gain coupled with a decrease in RAMAN gain. 


\section{Impact of Optical Booster to Enhance the Gain Bandwidth}

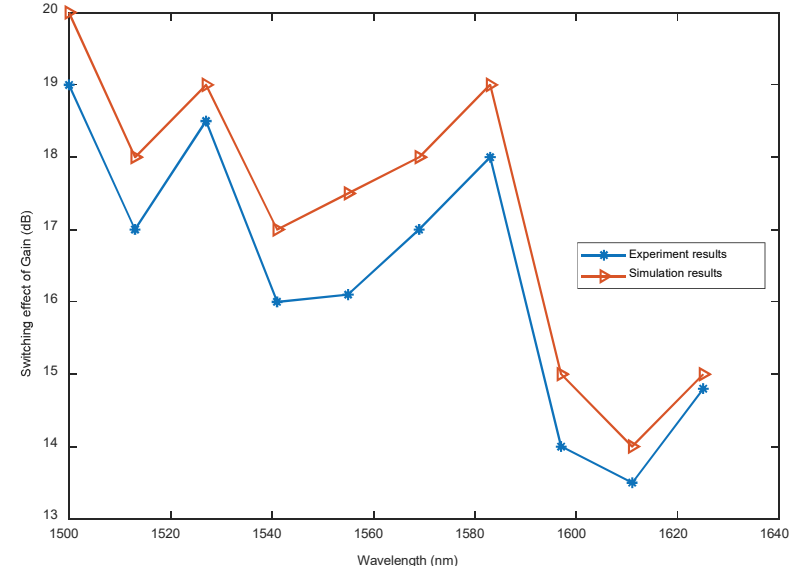

Fig. 4

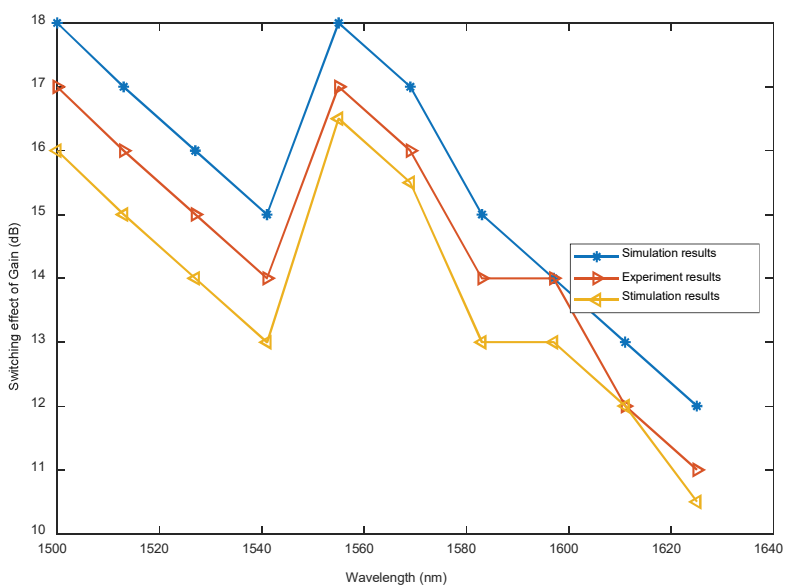

Fig. 5

Reconfiguration of independent variables leads to most favourable condition of the pumps (RAMAN pump tune at $1.6 \mathrm{w}$, para-metric pump at $0.38 \mathrm{w}$. For these set values, gain deviation of $5.4 \mathrm{~dB}$, on gain and off gain (maximum) is examined of $14 \mathrm{~dB}$. It was noted the polarizations of these signal impacts the gain compute in this region of powerful para-metric gain on a major level in comparison to the para-metric pump producing polarization. Two polarization controllers (PC), one each in PARA-METRIC pump and signal path control the relative polarization. The amplification is a two stage process. First HNLF segment amplifies the signals within the parametric gain system while as signal in RAMAN gain region get amplified by first HNLF segment. Both pumps (PARA-METRIC and RAMAN) amplify wavelength in region 1570-1580nm. By adjusting overlapping gain region, the gain variation can be minimized as well as optimized. Adjustments of pump with power helps to obtain the flat gain. The experimental data fares well the simulation that with relative error ( $\max$ ) at $12 \%$ with RAMAN region, while $10 \%$ in para-metric region of gain.

For Tandem \& Unison configuration, the noise figure can be expressed using -

$$
\mathrm{NF}_{\mathrm{S}}=2 \mathrm{P}_{\mathrm{PF}} / \eta \omega_{\mathrm{S}} \mathrm{BG}_{\mathrm{S}}+1 / \mathrm{G}_{\mathrm{s}}
$$

For single PARA-METRIC pump lowest NF values are 3.7, 3.8 and $4.2 \mathrm{~dB}$ from fig. 4 and fig. 5. Limited OPA pump source power, phase modulator and insertion loss compels for usage of EDFA. ASE is generated due to this and can be terminated by using a number of lower insertion filter.

Relative noise intensity also gets generated from SBS in the PARA-METRIC process. Signal with these (ASE and SBS) noise sources get onto second RAMAN amplification stage. Amplified spontaneous scattering by RAMAN process due to higher RAMAN pump (1.63W) is also a noise. Tandem $\mathrm{NF}$ value is $-34 \mathrm{~dB}$ greater than unison configuration value. Increased NF value of signal is due to increased ASE noise. This causes higher power at RAMAN pump. Usage of multiple lower power RAMAN pumps can solve the problem with a slight change in HNLF length, but the gain characteristics must be kept equivalent. while for unison configuration RAMAN region NF are lower than PARA-METRIC region value. ASE gets transferred, reduced by almost $6 \mathrm{~dB}$ from its RAMAN region peak while PARA-METRIC pump ASE is increased by the same amount. This transfer is controlled by para-metric pump wave-length. Selection of PARA-METRIC pump where RAMAN pair is maximum leads to large NF variations i.e. $7.3 \mathrm{~dB}-21 \mathrm{~dB}$. In regions of smaller RAMAN pair para-metric pump choice has reduced the NF variation 7.9dB to $13.7 \mathrm{~dB}$.

\section{THE STUDIES OF TIME DOMAIN}

For this time, study of the time domain, consolidate the configuration by keeping the RAMAN pump at 1.534 and the PARA-METRIC pump at 0.34W. BER performance (assuming Gaussian noise) was calculated by observing optical power over a range of varied wavelengths. Q-parameter is calculated as

$$
\mathrm{Q}=(-0.167+\sqrt{ }(0.028-\log ((\mathrm{BER} / 0.217)+3.92)
$$

Power penalty is observed at $1.58 \mathrm{~nm}$ wavelength due to increased NF and SBS noise. In unison configuration, the $15 \%$ of total of power of RAMAN is transferred to para-metric pump causing SBS noise. In Tandem configuration, SBS remains low due to no transfer of power between any pumps thus producing only $1 \mathrm{~dB}$ power penalty. 


\section{CONCLUSION OF THE EXPERIMENT}

Conclusion It turns out that by combining the PARAMETRIC and RAMAN process in one unit, practically and in principle, we can increase the BW by increasing the profit area to get RAM benefits. We come across the features like gain, BW, noise figure and error in bit rate for two nonlinear processes. Two different ways to achieve the flat gain bandwidth are Unison and Tandem. The mathematics shows that tuning the two pump, wavelength give improved gain flatness. Multiple RAMAN amplifier and proper HNLF length are used to reduce noise properties. In the RAMAN gain area (1545 nm-1570 nm), a negligible power penalty is produced for both Unison and Tandem. We configure that substantial increase can be seen in the gain region if we are provided with experimental flexibility. We mainly focus on the increase of gain BW and not on optimized parameters used in the combined RAMAN and Para-metric amplifiers.

\section{REFERENCES}

Pinto A M R, Frazão O, Santos J L, Lopez Amo M (2010) Multiwavelength fiber laser based on a photonic crystal fiber loop mirror with cooperative Rayleigh scattering: Appl. Phys. B, Lasers Opt. 99: 391-395.

Zhang L, Xu Y, Gao S, Saxena B, Chen L, Bao X (2018) Multiwavelength coherent Brillouin random fiber laser with ultrahigh optical signalto-noise ratio: IEEE J. Sel. Topics Quantum Electron 24: 0900308
Du X, Zhang H, V X, Wang X, Zhou P, Liu Z (2015) Multiwavelength Raman fiber laser based on polarization maintaining fiber loop mirror and random distributed feedback: Laser Phys. Lett. 12: 045106.

Sugavanam S, Tarasov N, Shu X, Churkin D V (2013) Narrow-band generation in random distributed feedback fiber laser: $0 p t$. Express 21: 16466-16472.

Pang M, Xie S, Bao X, Zhou D P, Lu Y, Chen L (2012) Rayleigh scattering-assisted narrow linewidth Brillouin lasing in cascaded fiber: opt. Lett. 37: 3129-3131.

Zhu T, Huang S, Shi L, Huang W, Liu M, Chiang K (2014) Rayleigh backscattering: A method to highly compress laser linewidth: Chin. Sci. Bull 59: 4631-4636.

Babin S A, Zlobina E A, Kablukov S I, Podivilov E V (2016) High-order random Raman lasing in a PM fiber with ultimate efficiency and narrow bandwidth: Sci. Rep. 6: 22625.

Zlobina E A, Kablukov S I, Babin S A (2015) Linearly polarized random fiber laser with ultimate efficiency: Opt. Lett. 40: 4074-4077.

Wu H, Wang Z N, Churkin D V, Vatnik I D, Fan M Q, Rao Y J (2015) Random distributed feedback Raman fiber laser with polarized pumping: Laser Phys. Lett. 12: 015101.

Xu J (2017) Near-diffraction-limited linearly polarized narrowlinewidth random fiber laser with record kilowatt output: Photon. Res. 5: 350-354.

Li T (2018) Power scaling of narrow-linewidth fiber amplifier seeded by Yb-doped random fiber laser: IEEE J. Sel. Topics Quantum Electron 24: 0903208.

Jin X, Lou Z, Zhang H, Xu J, Zhou P, Liu Z (2016) Random distributed feedback fiber laser at $2.1 \mu \mathrm{m}$ : Opt. Lett. 41: 4923-4926. 